\title{
A multi-object spectrograph using single-mode fibers with a coronagraph: progress towards laboratory results on the high- contrast testbed for segmented telescopes
}

Carl T. Coker, Jorge Llop Sayson, Stuart Shaklan, A. J. E. Riggs, Dimitri Mawet, et al.

Carl T. Coker, Jorge Llop Sayson, Stuart Shaklan, A. J. E. Riggs, Dimitri Mawet, Garreth Ruane, Nemanja Jovanovic, Dan Echeverri, "A multi-object spectrograph using single-mode fibers with a coronagraph: progress towards laboratory results on the high-contrast testbed for segmented telescopes," Proc. SPIE 11117, Techniques and Instrumentation for Detection of Exoplanets IX, 111171A (9 September 2019); doi: 10.1117/12.2528569

Event: SPIE Optical Engineering + Applications, 2019, San Diego, California, United States 


\title{
A Multi-Object Spectrograph Using Single-Mode Fibers With a Coronagraph: Progress Towards Laboratory Results on the High Contrast Testbed for Segmented Telescopes
}

\author{
Carl T. Coker ${ }^{\mathrm{a}, \mathrm{b}}$, Jorge Llop Sayson ${ }^{\mathrm{c}}$, Stuart Shaklan ${ }^{\mathrm{b}}$, A J E. Riggs ${ }^{\mathrm{b}}$, Dimitri Mawet $^{\mathrm{c}}$, \\ Garreth Ruane ${ }^{\mathrm{b}}$, Nemanja Jovanovic ${ }^{\mathrm{c}}$, and Dan Echeverric

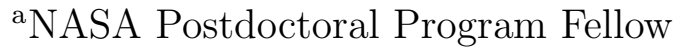 \\ b Jet Propulsion Laboratory, 4800 Oak Grove Drive, Pasadena, CA, USA \\ ${ }^{\mathrm{c}}$ California Institute of Technology, MC 249-17, 1200 E California Blvd, Pasadena, CA, USA
}

\begin{abstract}
Using single-mode fibers (SMFs) in the image plane of coronagraphs allows access to a new wavefront control regime. By using deformable mirrors to create a mismatch between the incoming starlight and the fiber mode, SMFs can serve as integral parts of the light suppression ability of the coronagraph. Previous promising simulation results show increased spectral bandwidth and throughput when using SMFs in a multi-object role, and previous laboratory results have shown increased light suppression using a single SMF. We present an update on efforts to combine a multi-core SMF with a high-resolution spectrograph on the High Contrast Testbed for Segmented Telescopes (HCST) at Caltech. We present our planned experimental design, as well as simulations of expected performance when controlling multiple fiber cores on HCST. We will test the potential increase in spectral bandpass and throughput resulting from the switch to SMFs, as well as the stability of the wavefront control solution.
\end{abstract}

Keywords:

\section{INTRODUCTION}

Directly imaging Earth-like planets around other stars is an extremely difficult task, yet it must be done if we are to spectrally characterize a large sample of these worlds. Doing so requires maintaining contrasts of $\sim 10^{-10}$ at sub-arcsecond separations for potentially hundreds of hours. ${ }^{1,2}$ One way of maintaining this contrast, allowing the faint planet to be imaged next to the comparatively overwhelmingly bright star, is a coronagraph. A large amount of research has been devoted to coronagraphy, and there exist several different architectures which offer the potential to directly image Earth-like exoplanets, many of which are approaching $10^{-10}$ contrast in the laboratory. ${ }^{3-18}$

Despite this progress, however, the spectral bandwidth and throughput of these designs remain limited, greatly lengthening the amount of time needed to obtain wide spectra for these worlds and fully characterize them. One possible solution to this problem is to use a single-mode fiber (SMF) in the final image plane. Recent simulations and laboratory results ${ }^{19-22}$ have shown that SMFs can be used as part of a wavefront control system while also offering improved spectral bandwidth and background-limited SNR ${ }^{23}$ However, like the coronagraphs themselves, they have so far not been demonstrated to achieve the simultaneous high contrasts and wide bandwidths that the simulations suggest are mathematically possible. We aim to change that, as well as demonstrate multi-fiber wavefront control at the same time. To that end, we will place a multicore SMF on the High Contrast Testbed for Segmented Telescopes (HCST) $)^{24,25}$ at Caltech and investigate the performance of a vector vortex focal plane mask when combined with SMFs.

In Section 2, we give a brief overview of SMF wavefront control. In Section 3, we describe our planned experimental design and the hardware we have already acquired. In Section 4, we discuss our simulations of expected laboratory performance, while in Section 5, we provide some brief conclusions.

Send correspondence to Carl T. Coker:

E-mail: carl.t.coker@jpl.nasa.gov, Telephone: +1 8183540104

Techniques and Instrumentation for Detection of Exoplanets IX, edited by Stuart B. Shaklan, Proc. of SPIE Vol. 11117, 111171A · C 2019 SPIE · CCC code: 0277-786X/19/\$21 - doi: 10.1117/12.2528569 


\section{SINGLE-MODE FIBER WAVEFRONT CONTROL}

The key innovation that has lead to this experiment and others like it such as Ref. 20 is the use of electric field conjugation (EFC) ${ }^{26}$ with a SMF. ${ }^{27}$ A SMF acts as a spatial filter, rejecting light which does not match the mode shape of the fiber. This property means that a SMF can be used as part of a wavefront control system $^{19,21-23,27,28}$ - in our case, instead of traditional EFC which attempts to use deformable mirrors (DMs) to null residual starlight in the image plane of a coronagraph, with fiber EFC, the output of the fiber is minimized instead. This means that the DMs are asked to create a mismatch between the residual starlight and the SMF mode. Simulations have shown that this has the potential to create large gains in contrast performance, ${ }^{19}$ spectral bandwidth, and even improve the SNR in background-limited observations. ${ }^{23}$ We seek to build on these results and replicate them in the lab, as described in the following sections.

\section{LABORATORY SETUP}

\subsection{Hardware Description}

We plan to use a multicore fiber with the HCST bench at Caltech, the current and planned optical layouts of which are shown in Figure 1 and 2, respectively. HCST is a currently operational in-air coronagraphic testbed ${ }^{24}$ with a boradband tunable light source and a single 34x34-actuator Boston Micromachines Kilo-DM to provide wavefront control. It can use a variety of coronagraph masks, and is currently configured for transmissive focal plane masks. At present, light from the coronagraph is imaged onto an Andor Neo 5.5, but we plan to build a fiber injection unit (FIU) to allow the use of SMFs on the bench. Of note is that the red limit of our tunable filter (an NKT Photonics SuperK VARIA) is $840 \mathrm{~nm}$; although the supercontinuum source and the rest of our optics can go redder, we would need another solution to produce narrowband light for wavefront control. This

effectively sets the red limit for our experiment. For a more detailed description of the current state of the bench and recent wavefront control results, see Ref. 25.

Figure 3 shows a picture of our multicore fiber. It is a 7-core Chiral Photonics PROFA 2D which has a fused end with the 7 cores arranged in a filled hex pattern and 7 pigtails on the other end. The pigtails are S630-HP optical fiber, placing the single-mode cutoff of the total fiber in the range of $550-600 \mathrm{~nm}$. The core/channel spacing on the multicore end is $37 \mu \mathrm{m}$, and the diameter of each core is $3.5 \mu \mathrm{m}$; the mode field diameters are $\sim 5.5 \mu \mathrm{m}$ at $730 \mathrm{~nm}$. We will ultimately bring the pigtails back together into a V-groove assembly and then image and magnify the fiber tips onto our Andor Neo camera.

\subsection{Experimental Design}

Once we have successfully aligned the multicore fiber and can image the output onto the Andor Neo, we can begin our experiment. We will first see how good our contrast is in narrowband light to verify that wavefront control is working, and then move on to the broadband portion of the experiment, where our goal is to realize as much of the performance gains shown in Ref. 23 as possible. The experiment itself is therefore simple: push the bandwidth as wide as possible while still maintaining better than $10^{-8}$ contrast, which is the best that HCST has so far been able to achieve in narrowband light in imaging mode. ${ }^{25}$ Because we will only be using a single 34x34-actuator DM, we may not be able to fit all 7 cores in the correctable field of view and still control them/ensure they don't overlap in spatial frequency. The number of DMs is important because EFC cannot, in general, find a solution for a full $360^{\circ}$ dark hole with only one DM because of the overlap of spatial frequencies - a DM shape that improves the contrast on one side of the field tends to make the other side worse, and vice versa. This is a problem with all linear wavefront controllers. Nevertheless, we will be able to correct at least $3-4$ cores at a time, demonstrating the principle of controlling multiple SMFs at arbitrary positions in the focal plane.

\section{EXPECTED LABORATORY PERFORMANCE}

\subsection{Simulation Design}

As with the simulations in Ref. 23, we used the $\mathrm{FALCO}^{29}$ software package to perform the wavefront sensing and control. We approximated the true multicore fiber mode as individual single fibers overlaid atop one another, 


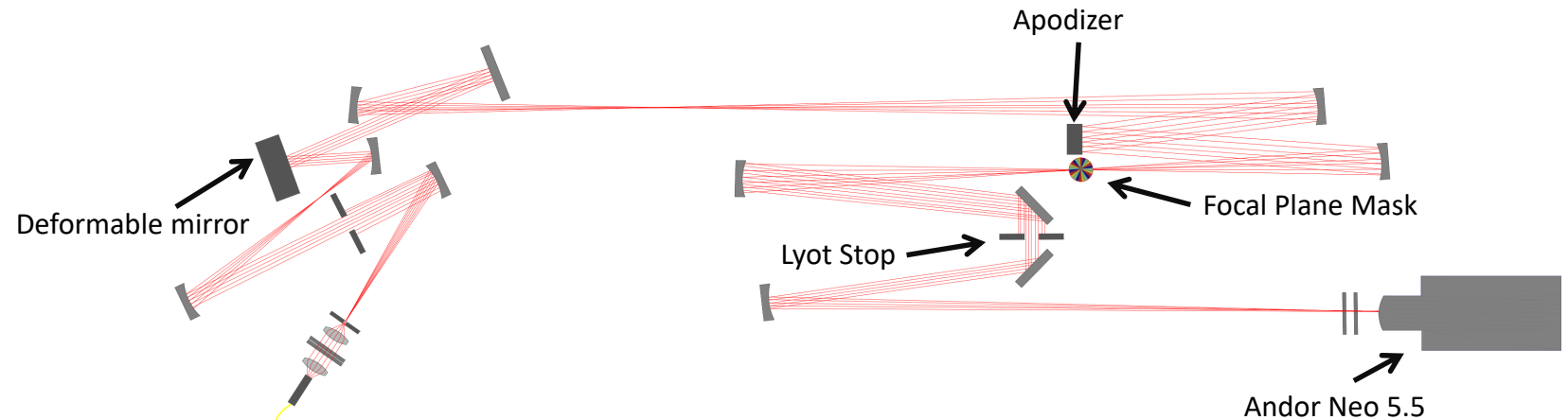

Source:

NKT Photonics SuperK

EXTREME and VARIA

Figure 1. Current layout of the HCST bench.

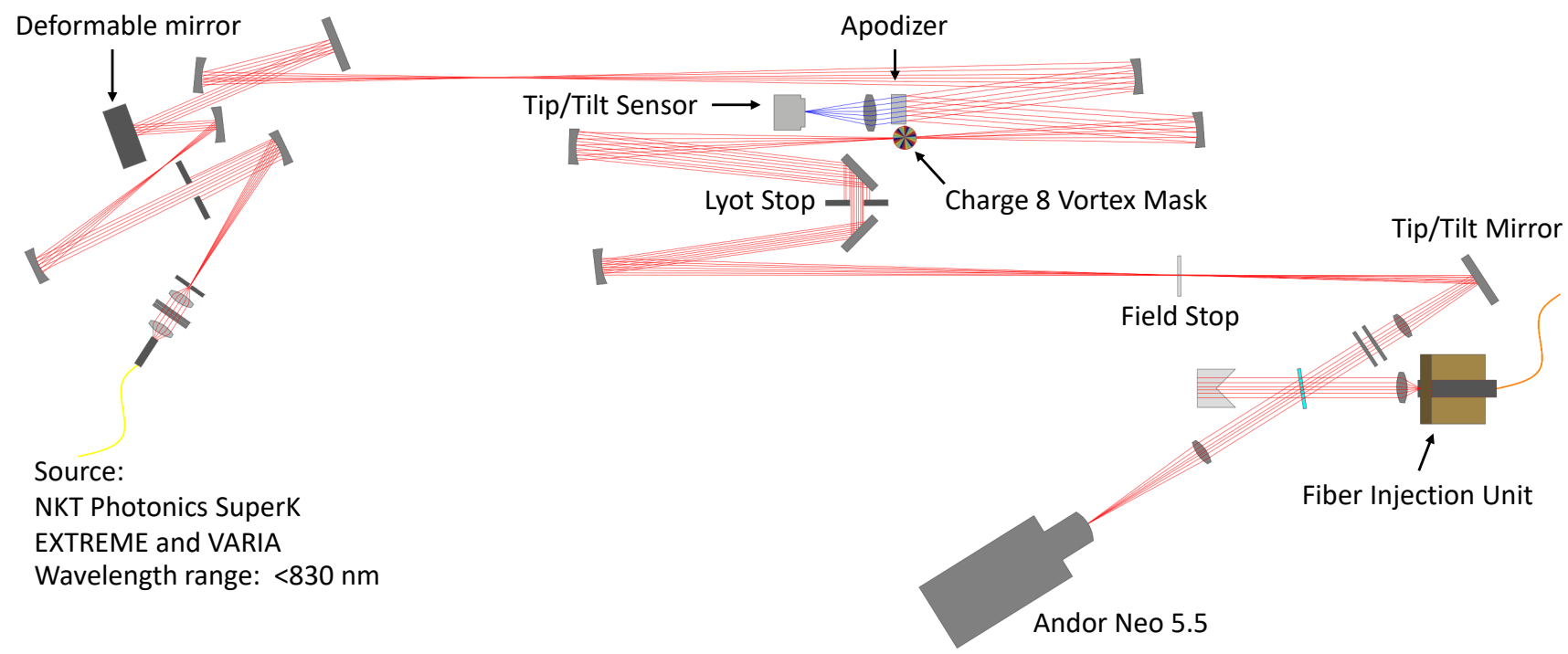

Figure 2. Planned layout of the HCST bench with the fiber injection unit.

Proc. of SPIE Vol. 11117 111171A-3 


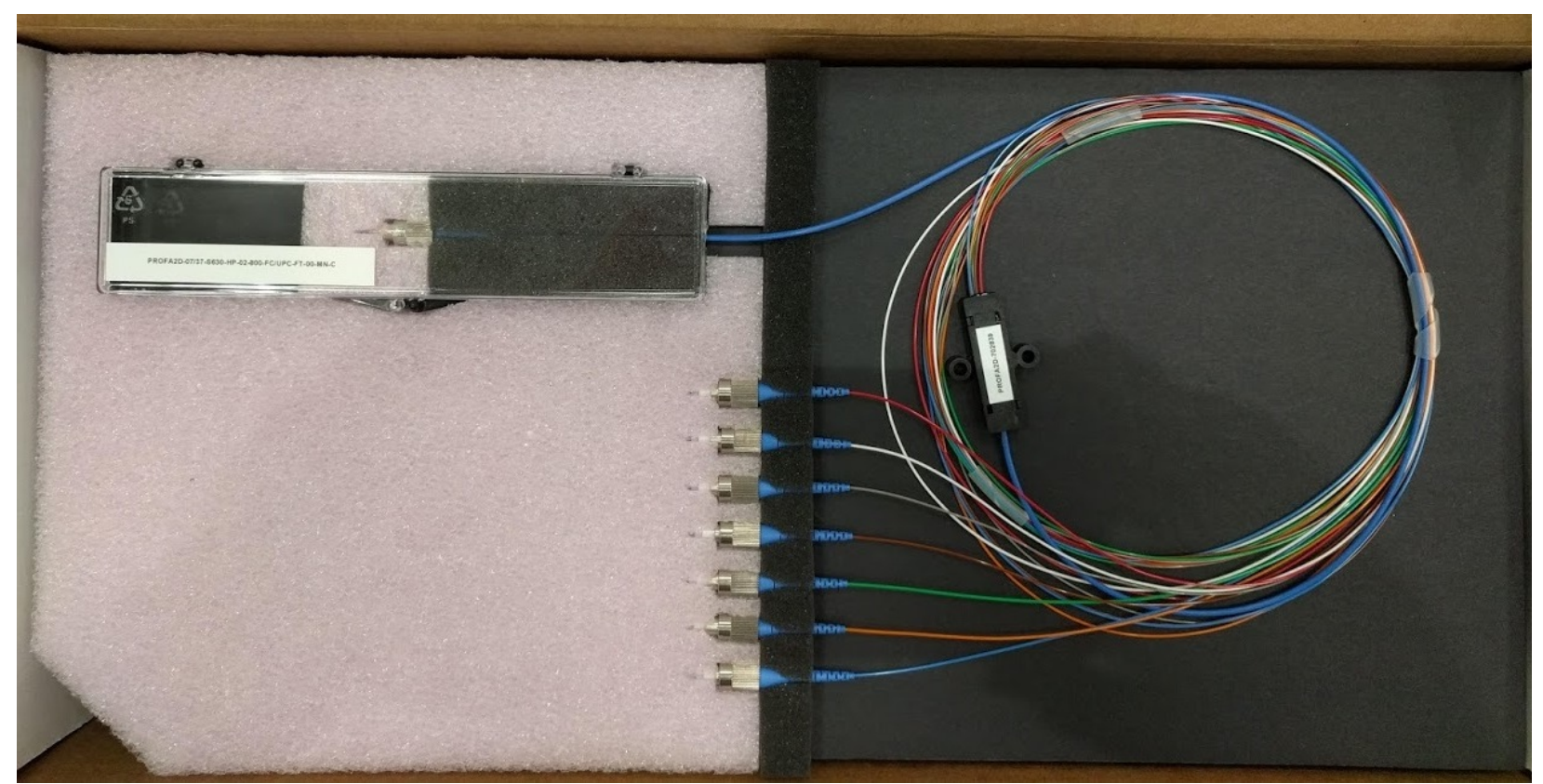

Figure 3. Picture of our multicore fiber, a PROFA 2D from Chiral Photonics. The multicore end has the 7 cores arranged in a hex pattern. The cores are then broken out into individual pigtails of S630-HP optical fiber. The core diameter of $\mathrm{S} 630-\mathrm{HP}$ is $3.5 \mu \mathrm{m}$, and the channel spacing of the multicore face is $37 \mu \mathrm{m}$. With extremely low crosstalk between cores of $\sim-60 \mathrm{~dB}$ or lower, the fiber itself should not place any limit on our performance.

with the output electric fields treated as coherent and summed; as the crosstalk between the cores is so low $(\sim-60 \mathrm{~dB}$ at $730 \mathrm{~nm})$, this is a very close approximation of the electric field in the single-mode cores themselves. For the purposes of the simulation, we used just three fiber cores arranged in an equilateral triangle centered on the optical axis of the system; we will probably not be able to fit all 7 cores into the correctable field of view, especially when using only one DM. This arrangement is shown in Figure 4. The spacing of the fiber cores in the simulation was $10.48 \lambda_{0} / D$, which matches the $37 \mu \mathrm{m}$ core spacing of the actual fiber when the $15.2 \mathrm{~mm}$ diameter beam from the Lyot stop is imaged onto the fiber using at $\mathrm{f} / 5$.

For the DM, we used $132 \times 32$ actuator DM. This is slightly smaller than the actual actuator count of the HCST DM (which is $34 \times 34$ ), but as the three cores are well away from the edge of the controllable zone in either case, it makes no real difference. We set the minimum step size for the DM actuators to be $300 \mathrm{pm}$, our rough internal estimate of the step corresponding to the least significant bit (LSB) of our current DM electronics. This setting is extremely important, because as shown in the next section, it may ultimately be the primary limitation on our achievable contrast.

We simulated an achromatic charge 8 vortex mask operating on a hexagonally segmented off-axis pupil. No real mask is perfectly achromatic, but we believe ours to have manageable leakage over a relatively broad band (though we have performed no formal measurement of this). Even so, the inherent chromaticity and retardance errors present in the mask are likely to set the ultimate limit on our achievable bandwidth at our contrast floor. In the simulations, we control a $40 \%$ bandwidth centered on $690 \mathrm{~nm}$. This is halfway between our fiber singlemode cutoff wavelength of $\sim 550 \mathrm{~nm}$ and the $830 \mathrm{~nm}$ red cutoff of our VARIA, beyond which it becomes much less convenient to do wavefront control. We control 12 separate wavelengths in the simulations; we have found that $3-3.5 \%$ bandwidth per controlled wavelength is roughly the limit of what can be done while still hitting the contrast floor.

For estimating the electric field, we compared the performance of pairwise probing ${ }^{30}$ and assuming perfect knowledge. For the pairwise probing, we used 3 pairs of probes evenly separated in phase. The probe shape for each pair was a sum of three sinusoids on the DM placed so that the maxima of the focal plane intensity patterns were close to the centers of the fibers. This and our $300 \mathrm{pm}$ minimum DM step size were our only deviations 


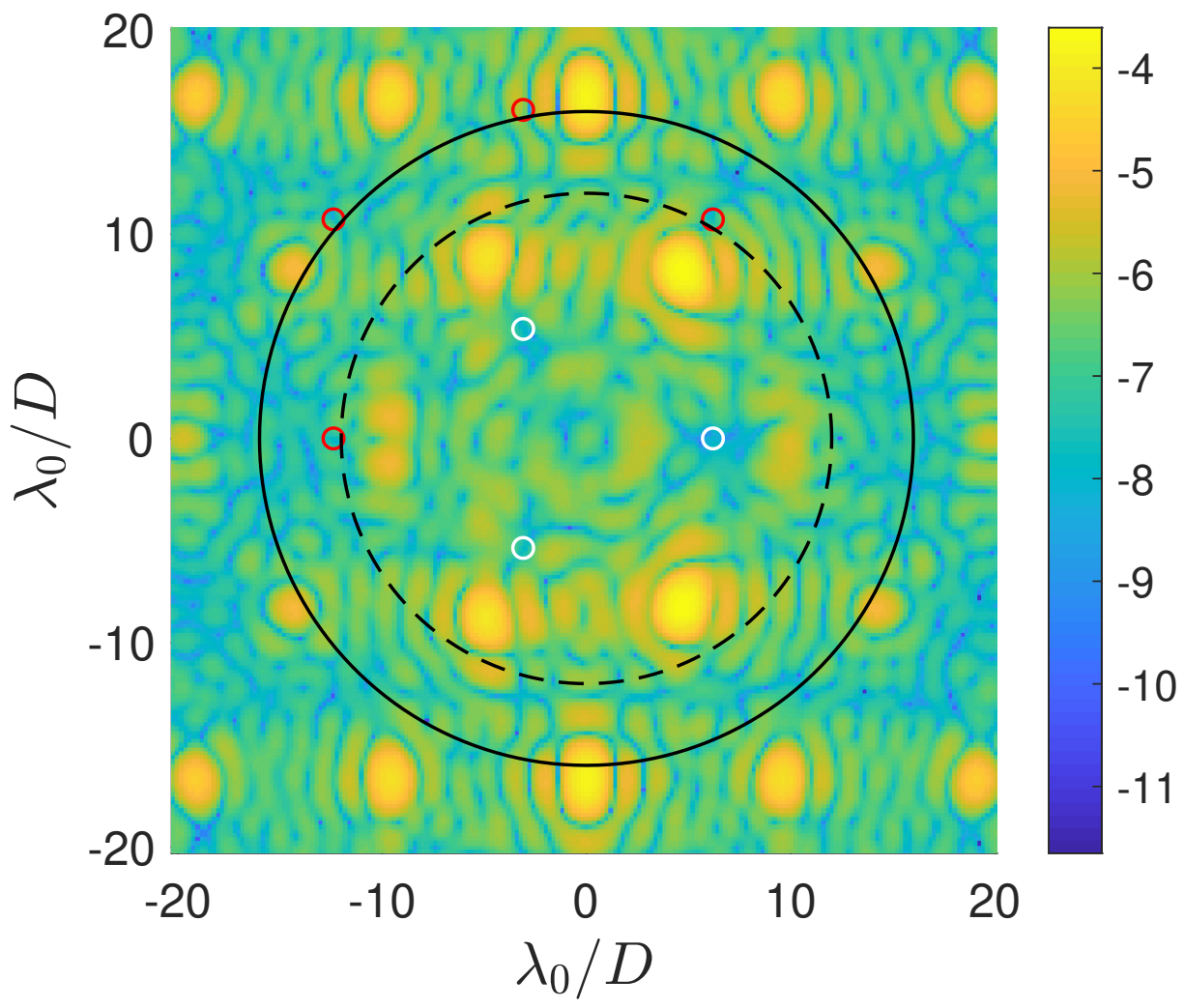

Figure 4. Normalized intensity map in the coronagraph image plane for the performance simulations described in this Section. The small white and red $0.507 \lambda_{0} / D$-radius circles show the locations of the 7 fiber cores; the 3 white cores were wavefront controlled in our simulations, while the red ones were not. The large solid black circle is the outer $16 \lambda_{0} / D$ control radius of our simulated DM, while the dashed black circle at $12 \lambda_{0} / D$ shows where the wavefront control on HCST begins to degrade in our experience. For that reason, we would like to be able to put as many cores inside that radius as possible.

from perfection. We did not assume any noise, optical surface errors, jitter from the fact that our testbed is in air, reflection losses, stray light, etc. Therefore, the proceeding results represent the best case scenario for our experiment.

\subsection{Simulation Results}

Figure 5 shows the results of our main performance simulations. The average contrast level achieved in the fibers at $(-3.0, \pm 5.4) \lambda_{0} / D$ is $1-2 \times 10^{-9}$ across the $550-830 \mathrm{~nm}$ wavelength range. We suspect that the excellent performance of the fiber core at $(6.2,0) \lambda_{0} / D$ is mostly due to luck - i.e., that it happened to fall on a particularly faint part of the field. Therefore, we expect the performance to be more in line with the other two fiber cores simulated. Due to the lack of leakage or other defects in the modeled vortex mask, we are able maintain a very wide $40 \%$ bandwidth (and probably much more, as in our previous simulation paper ${ }^{23}$ ). The throughput curves for this simulation are shown in Figure 6. The throughput of each fiber is nearly identical as they are all at the same distance from the center of the field. The slope is mainly due to how the throughput of a vortex mask changes with distance from the center as a function of $\lambda / D$, with an additional effect from the mismatch in how the fiber mode and PSF size scale with wavelength.

The largest limitation on our achievable contrast is likely the LSB of our DM electronics, which in large part sets the minimum step size for each actuator. The HCST DM currently has 14-bit electronics with an estimated minimum step size of $\sim 300 \mathrm{pm}$, setting a hard limit on how low we can go. Figure 7 shows the results of a second set of simulations identical to those presented in Figure 5, except that there is no minimum step size for 

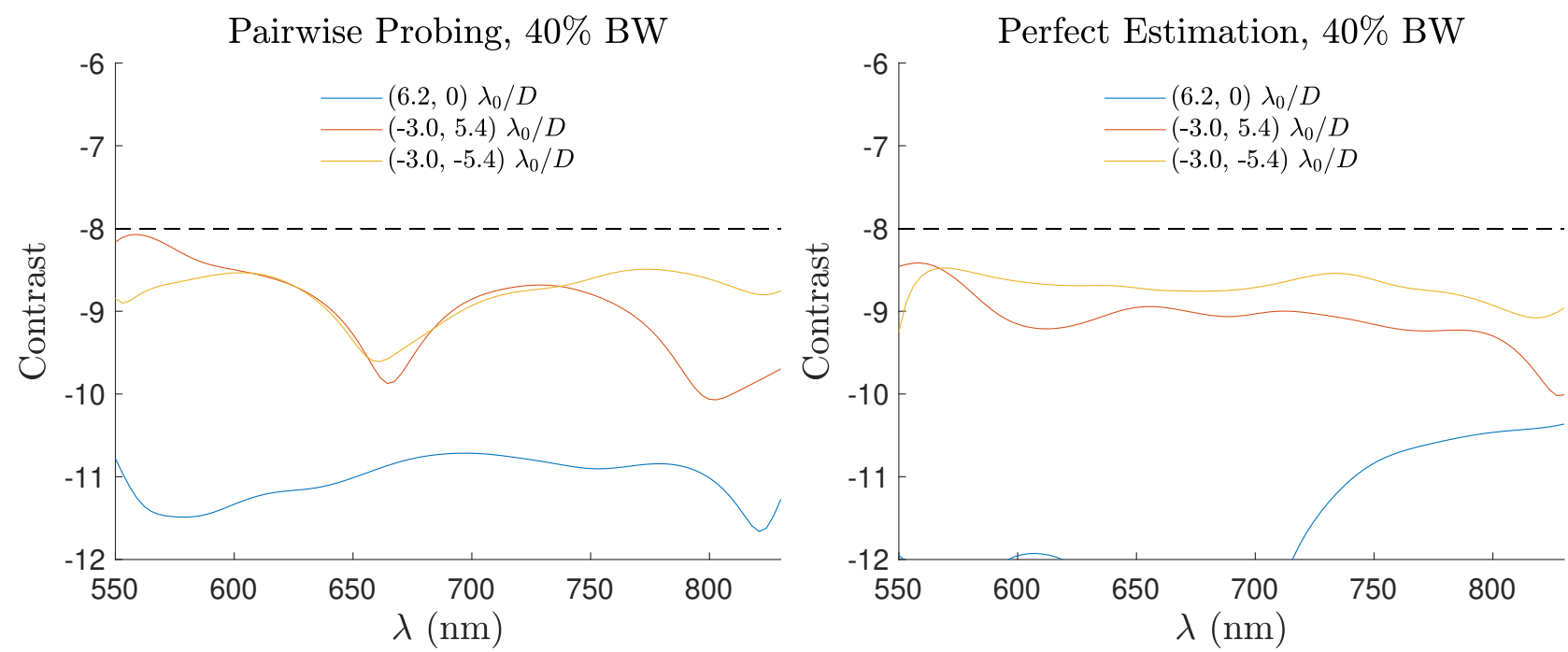

Figure 5. Contrast vs. wavelength for our performance simulations of the multicore fiber on the HCST bench. The left panel shows our results using pairwise probing to estimate the electric field, while the right panel shows our results using perfect knowledge of the field. The fact that pairwise probing shows the same average contrast as the perfect estimator is encouraging for our ability to actually achieve the $1-2 \times 10^{-9}$ contrast floor shown here. Each line represents one fiber core; the legends show the $(\mathrm{x}, \mathrm{y})$ position of each core. Based on these simulations, we expect the fibers to achieve $3-10$ times better contrast than the current HCST imaging mode floor.

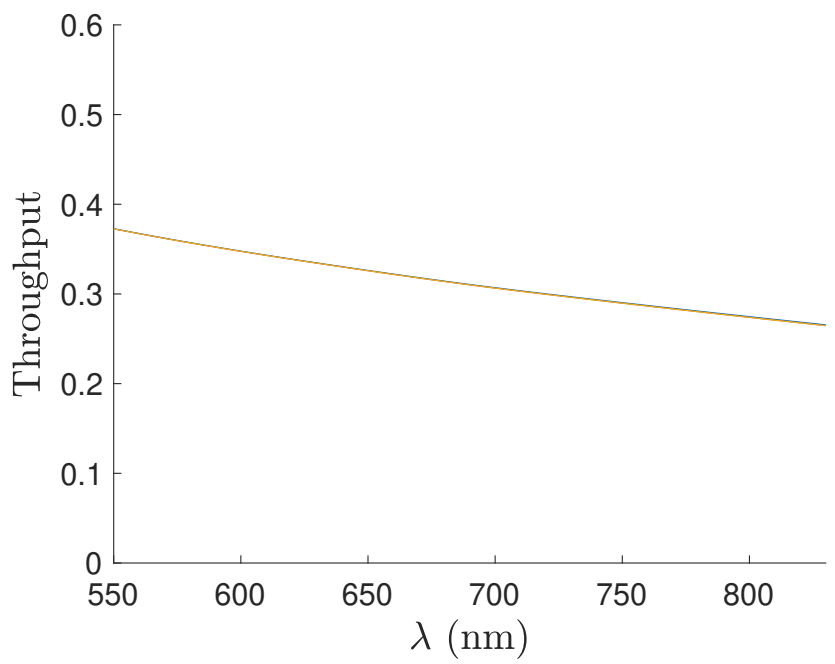

Figure 6. Throughput vs. wavelength for our HCST multicore fiber performance simulations. The three curves for the individual fiber cores lie right on top of one another because each core is the same distance from the center of the field. The throughput shown here is in line with our previous simulations results from Ref. 23.

the DM actuators. An improvement of at least an order of magnitude in contrast is evident. Therefore, if we do manage to hit the LSB floor, better contrast may be possible with better electronics.

\section{CONCLUSIONS}

In this paper, we have presented an update on our progress towards demonstrating the use of multiple SMFs for coronagraphy and exoplanet characterization. We will use a multicore fiber in conjunction with the HCST testbed at Caltech to investigate the performance of this configuration; we aim to demonstrate the extended bandwidth and high contrast results shown in previous simulations. We will build the HCST FIU over the following months, verify its performance with a single fiber, and subsequently begin our multicore testing. Our 

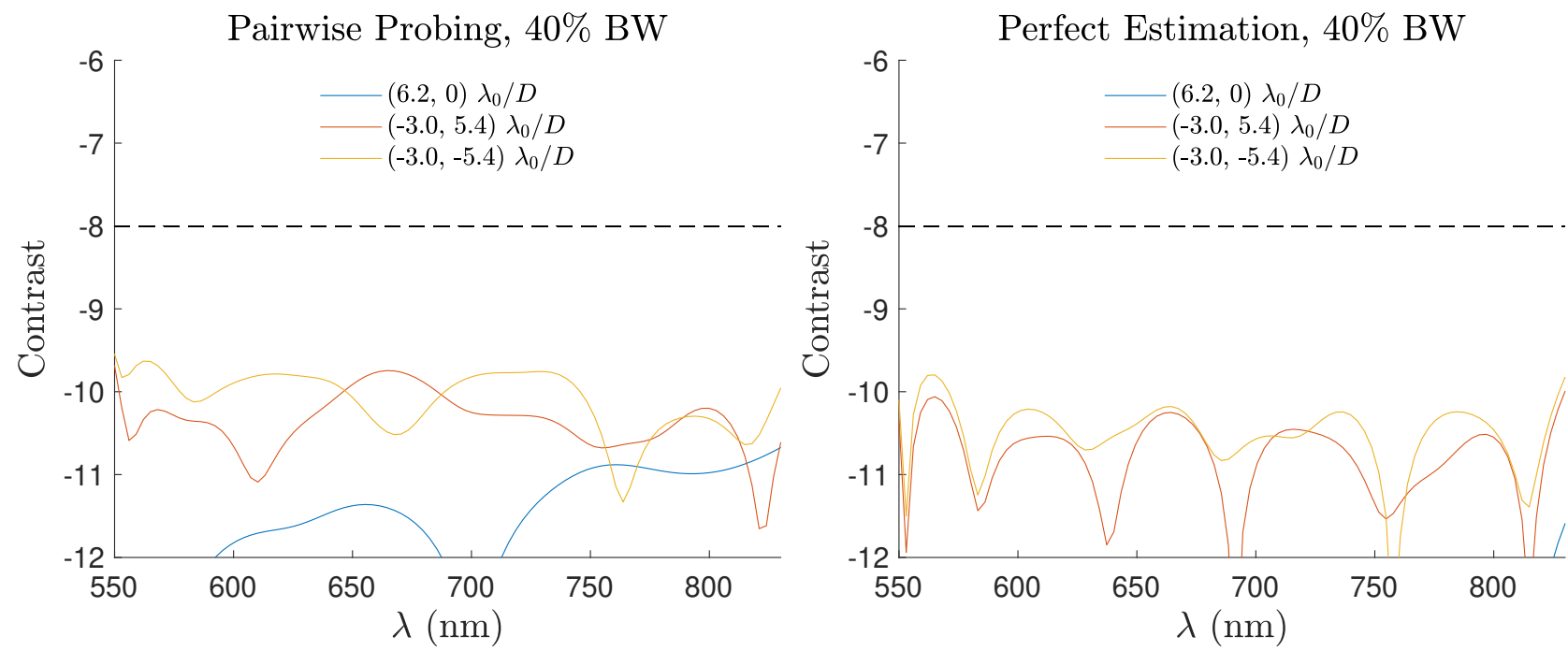

Figure 7. Contrast vs. wavelength for perfromance simulations of the multicore fiber on HCST with no LSB/DM minimum step size. The contrast improves by at least an order of magnitude over that shown in Figure 5, showing that the DM LSB is an important factor in system performance at these high contrast ratios. Otherwise as Figure 5.

best-case contrast with current equipment is $1-2 \times 10^{-9}$ over a $40 \%$ spectral bandwidth in air, with the biggest limiting factors after that being the LSB of our DM electronics and leakage/defects in our current vortex mask. Demonstrating improved contrast and bandwidth through SMFs would represent a large leap forward in coronagraph performance, especially for spectral characterization with future space telescopes.

\section{ACKNOWLEDGMENTS}

This work was performed at the Jet Propulsion Laboratory, California Institute of Technology, under a contract with the National Aeronautics and Space Administration. This work was supported by an appointment to the NASA Postdoctoral Program at the Jet Propulsion Laboratory, administered by the Universities Space Research Association under contract with NASA. Copyright 2019 California Institute of Technology. Government Sponsorship acknowledged. All rights reserved.

\section{REFERENCES}

[1] Wang, J., Mawet, D., Ruane, G., Hu, R., and Benneke, B., "Observing Exoplanets with High Dispersion Coronagraphy. I. The Scientific Potential of Current and Next-generation Large Ground and Space Telescopes," AJ 153, 183 (Apr 2017).

[2] Wang, J., Mawet, D., Hu, R., Ruane, G., Delorme, J.-R., and Klimovich, N., "Baseline requirements for detecting biosignatures with the HabEx and LUVOIR mission concepts," Journal of Astronomical Telescopes, Instruments, and Systems 4, 035001 (Jul 2018).

[3] Soummer, R., "Apodized Pupil Lyot Coronagraphs for Arbitrary Telescope Apertures," ApJ 618, L161L164 (Jan 2005).

[4] Foo, G., Palacios, D. M., and Swartzland er, Grover A., J., "Optical vortex coronagraph," Optics Letters 30, 3308-3310 (Dec 2005).

[5] Mawet, D., Riaud, P., Absil, O., and Surdej, J., "Annular Groove Phase Mask Coronagraph," ApJ 633, 1191-1200 (Nov 2005).

[6] Kasdin, N. J., Vanderbei, R. J., Spergel, D. N., and Littman, M. G., "Extrasolar Planet Finding via Optimal Apodized-Pupil and Shaped-Pupil Coronagraphs," ApJ 582, 1147-1161 (Jan 2003).

[7] Guyon, O., Pluzhnik, E. A., Galicher, R., Martinache, F., Ridgway, S. T., and Woodruff, R. A., "Exoplanet Imaging with a Phase-induced Amplitude Apodization Coronagraph. I. Principle," ApJ 622, 744-758 (Mar 2005). 
[8] Kuchner, M. J. and Traub, W. A., "A Coronagraph with a Band-limited Mask for Finding Terrestrial Planets," ApJ 570, 900-908 (May 2002).

[9] Trauger, J., Moody, D., Gordon, B., Krist, J., and Mawet, D., "A hybrid Lyot coronagraph for the direct imaging and spectroscopy of exoplanet systems: recent results and prospects," in [Techniques and Instrumentation for Detection of Exoplanets V], Society of Photo-Optical Instrumentation Engineers (SPIE) Conference Series 8151, 81510G (Oct 2011).

[10] Coker, C. T., Ruane, G., Riggs, A. J. E., Sidick, E., Seo, B.-J., Kern, B., Marx, D., and Shaklan, S., "Fast linearized coronagraph optimizer (FALCO) III: optimization of key coronagraph design parameters," in [Society of Photo-Optical Instrumentation Engineers (SPIE) Conference Series], 10698, 1069851 (Aug 2018).

[11] Ruane, G., Riggs, A., Coker, C. T., Shaklan, S. B., Sidick, E., Mawet, D., Jewell, J., Balasubramanian, K., and Stark, C. C., "Fast linearized coronagraph optimizer (FALCO) IV: coronagraph design survey for obstructed and segmented apertures," in [Space Telescopes and Instrumentation 2018: Optical, Infrared, and Millimeter Wave], Society of Photo-Optical Instrumentation Engineers (SPIE) Conference Series 10698, 106984U (Aug 2018).

[12] N'Diaye, M., Soummer, R., Pueyo, L., Carlotti, A., Stark, C. C., and Perrin, M. D., "Apodized Pupil Lyot Coronagraphs for Arbitrary Apertures. V. Hybrid Shaped Pupil Designs for Imaging Earth-like planets with Future Space Observatories," ApJ 818, 163 (Feb 2016).

[13] Ruane, G., Jewell, J., Mawet, D., Pueyo, L., and Shaklan, S., "Apodized vortex coronagraph designs for segmented aperture telescopes," in [Advances in Optical and Mechanical Technologies for Telescopes and Instrumentation II], Society of Photo-Optical Instrumentation Engineers (SPIE) Conference Series 9912, 99122L (Jul 2016).

[14] Zimmerman, N. T., N'Diaye, M., St. Laurent, K. E., Soummer, R., Pueyo, L., Stark, C. C., Sivaramakrishnan, A., Perrin, M., Vanderbei, R. J., Kasdin, N. J., Shaklan, S., and Carlotti, A., "Lyot coronagraph design study for large, segmented space telescope apertures," in [Space Telescopes and Instrumentation 2016: Optical, Infrared, and Millimeter Wave], Society of Photo-Optical Instrumentation Engineers (SPIE) Conference Series 9904, 99041Y (Jul 2016).

[15] Fogarty, K., Mazoyer, J., St. Laurent, K., Soummer, R., N’Diaye, M., Stark, C., and Pueyo, L., "Optimal deformable mirror and pupil apodization combinations for apodized pupil Lyot coronagraphs with obstructed pupils," in [Society of Photo-Optical Instrumentation Engineers (SPIE) Conference Series], 10698, 106981J (Aug 2018).

[16] Kern, B., Guyon, O., Kuhnert, A., Niessner, A., Martinache, F., and Balasubramanian, K., "Laboratory demonstration of Phase Induced Amplitude Apodization (PIAA) coronagraph with better than $10^{-9}$ contrast," in [Techniques and Instrumentation for Detection of Exoplanets VI], Society of Photo-Optical Instrumentation Engineers (SPIE) Conference Series 8864, 88640R (Sep 2013).

[17] Serabyn, G., Trauger, J., Moody, D., Mawet, D., Krist, J., and Kern, B., "Technology milestone \#1 report: Vortex coronagraph technology," (2014).

[18] Trauger, J. T. and Traub, W. A., "A laboratory demonstration of the capability to image an Earth-like extrasolar planet," Nature 446, 771-773 (Apr 2007).

[19] Mawet, D., Ruane, G., Xuan, W., Echeverri, D., Klimovich, N., Randolph, M., Fucik, J., Wallace, J. K., Wang, J., Vasisht, G., Dekany, R., Mennesson, B., Choquet, E., Delorme, J. R., and Serabyn, E., "Observing Exoplanets with High-dispersion Coronagraphy. II. Demonstration of an Active Single-mode Fiber Injection Unit," ApJ 838, 92 (Apr 2017).

[20] Llop Sayson, J., Ruane, G., Mawet, D., Jovanovic, N., Calvin, B., Levraud, N., Roberson, M., Delorme, J.-R., Echeverri, D., Klimovich, N., and Xin, Y., "Demonstration of an electric field conjugation algorithm for improved starlight rejection through a single mode optical fiber," Journal of Astronomical Telescopes, Instruments, and Systems 5, 019004 (Jan 2019).

[21] Por, E. H. and Haffert, S. Y., "The Single-mode Complex Amplitude Refinement (SCAR) coronagraph: I. Concept, theory and design," arXiv e-prints, arXiv:1803.10691 (Mar 2018).

[22] Haffert, S. Y., Por, E. H., Keller, C. U., Kenworthy, M. A., Doelman, D. S., Snik, F., and Escuti, M. J., "The Single-mode Complex Amplitude Refinement (SCAR) coronagraph: II. Lab verification, and toward the characterization of Proxima b," arXiv e-prints , arXiv:1803.10693 (Mar 2018). 
[23] Coker, C. T., Shaklan, S. B., Riggs, A. J. E., and Ruane, G., "Simulations of a High-Contrast SingleMode Fiber Coronagraphic Multi-Object Spectrograph for Future Space Telescopes," arXiv e-prints , arXiv:1907.03921 (Jul 2019).

[24] Jovanovic, N., Ruane, G., Echeverri, D., Delorme, J. R., Mawet, D., Fucik, J., Wallace, J. K., Coker, C., Delacroix, A., Levraud, N., Llop Sayson, J. D., Wang, J., Riddle, R., and Millar-Blanchaer, M. A., "Highcontrast spectroscopy testbed for Segmented Telescopes: instrument overview and development progress," in [Proc. SPIE], Society of Photo-Optical Instrumentation Engineers (SPIE) Conference Series 10702, 107024E (Jul 2018).

[25] Llop Sayson, J., Ruane, G., Jovanovic, N., Mawet, D., Echeverri, D., Riggs, A. J. E., Coker, C. T., Morrissey, G., and Sun, H., "The high-contrast spectroscopy testbed for segmented telescopes (HCST): new wavefront control demonstrations," in [Society of Photo-Optical Instrumentation Engineers (SPIE) Conference Series], 11117, 11117-54 (Aug 2019).

[26] Give'on, A., Kern, B., Shaklan, S., Moody, D. C., and Pueyo, L., "Broadband wavefront correction algorithm for high-contrast imaging systems," in [Astronomical Adaptive Optics Systems and Applications III], Society of Photo-Optical Instrumentation Engineers (SPIE) Conference Series 6691, 66910A (Sep 2007).

[27] Llop Sayson, J., Mawet, D., Ruane, G., Delorme, J.-R., Echeverri, D., Jovanovic, N., Klimovich, N., and Xin, Y., "Wavefront control for minimization of speckle coupling into a fiber injection unit based on the electric field conjugation algorithm," in [Society of Photo-Optical Instrumentation Engineers (SPIE) Conference Series], 10703, 1070372 (Jul 2018).

[28] Shaklan, S. and Roddier, F., "Coupling starlight into single-mode fiber optics," Appl. Opt. 27, 2334-2338 (Jun 1988).

[29] Riggs, A. J. E., Ruane, G., Sidick, E., Coker, C., Kern, B. D., and Shaklan, S. B., "Fast linearized coronagraph optimizer (FALCO) I: a software toolbox for rapid coronagraphic design and wavefront correction," in [Society of Photo-Optical Instrumentation Engineers (SPIE) Conference Series], 10698, 106982V (Aug 2018).

[30] Give'on, A., Kern, B. D., and Shaklan, S., "Pair-wise, deformable mirror, image plane-based diversity electric field estimation for high contrast coronagraphy," in [Proc. SPIE], Society of Photo-Optical Instrumentation Engineers (SPIE) Conference Series 8151, 815110 (Oct 2011). 